\title{
SÍNTESIS DE HIDROGELES CONMUTABLES A PARTIR DE N-ISOPROPILACRILAMIDA, MACROMONÓMEROS DE 2-OXAZOLINAS Y LAPONITA
}

\author{
Juan Carlos Rueda*a, María Malásquez ${ }^{\mathrm{a}}$, Lesly Lagos ${ }^{\mathrm{a}}$, Hartmut Komber ${ }^{\mathrm{b}}$, \\ Stefan Zschoche ${ }^{\mathrm{b}}$, Brigitte Voit ${ }^{\mathrm{b}}$
}

\begin{abstract}
RESUMEN
Fueron elaborados nuevos hidrogeles a partir de la copolimerización radicalar, en medio acuoso, de la $\mathrm{N}$-isopropilacrilamida (NiPAAm) y macromonómeros de 2-oxazolinas en presencia de la arcilla Laponita XLS (Hectorita sintética). Los macromonómeros utilizados, M1 y M2, fueron copolímeros estadísticos de 2-(2-metoxicarboniletil)- y 2-metil-2oxazolina, con grados de polimerización de 20 y 24 unidades y un contenido de 23 y $49 \%$ molar del monómero 2-(2-metoxicarboniletil)-2-oxazolina, respectivamente y contuvieron un grupo vinilo en el extremo de la cadena polimérica. La síntesis de los hidrogeles fue llevada a cabo utilizando el sistema de iniciación: persulfato de amonio (APS) y N, N, N, N-tetrametiletilendiamina (TEMED) y como entrecruzador la Laponita XLS. Los grupos ésteres de los hidrogeles sintetizados fueron hidrolizados in situ para obtener grupos ácido carboxílicos y finalmente se obtuvieron hidrogeles con buena consistencia mecánica, transparencia y sensibilidad a la temperatura y al $\mathrm{pH}$. Los hidrogeles fueron caracterizados estructuralmente vía resonancia magnética nuclear $(\mathrm{RMN})$, y también por su capacidad de absorción de agua, estabilidad mecánica y su sensibilidad a la temperatura y al $\mathrm{pH}$. La distribución del contenido de las partículas de Laponita dentro del hidrogel fue homogénea y fue determinada mediante microscopía electrónica de transmisión (TEM). Los hidrogeles mostraron sensibilidad a la temperatura debido a su contenido de segmentos de poli(Nisopropilacrilamida) y sensibilidad al $\mathrm{pH}$, por su contenido de grupos ácido carboxílicos.
\end{abstract}

Palabras clave: N-isopropilacrilamida, macromonómero, 2-oxazolinas, Laponita XLS, hidrogeles, sensibilidad térmica.

a Pontificia Universidad Católica del Perú, jrueda@pucp.edu.pe

${ }^{\mathrm{b}}$ Leibniz Institute for Polymer Research Dresden, Germany 


\title{
SYNTHESIS OF CONMUTABLES HYDROGELS BASED ON N-ISOPROPYLACRYLAMIDE, 2-OXAZOLINE MACROMONOMERS AND LAPONITE
}

\begin{abstract}
New hydrogels were synthesized based on free radical polymerization of $\mathrm{N}$-isopropylacrylamide (NiPAAm) and macromonomers of 2-oxazolines in presence of clay Laponite XLS (synthetic hectorite). The used macromonomers were statistical copolymers of 2-(2-methoxycarbonylethyl)- and 2-methyl-2-oxazoline, with a polymerization degree of 20 and 24 units and content of 23 and 49 mol-\% of 2-(2-methoxycarbonylethyl)-2-oxazoline, respectively, and had a vinyl group at the chain end. The hydrogel synthesis was carried out using ammonium persulfate (APS) and N,N,N,N-tetramethylethylendiamine (TEMED) as initiating system and Laponite XLS as crosslinker. After polymerization, the esters groups of the obtained hydrogels were hydrolized in situ to obtain carboxylic acids groups. Finally, hydrogels were obtained with high mechanical stability, transparency, and responsivity to temperature and $\mathrm{pH}$. The hydrogels were characterized structurally via nuclear magnetic resonance (NMR) and in addition, water absorption capacity, mechanical stability and temperature and $\mathrm{pH}$-responsivity were determined. The distribution of Laponite particles inside hydrogels was homogenous as confirmed by transmission electronic microscopy (TEM). The hydrogels showed temperature responsivity due to the poly (NiPAAm) segments and $\mathrm{pH}$-responsivity due to the carboxylic acids content.
\end{abstract}

Key words: N-isopropylacrylamide, macromonomer, 2-oxazolines, Laponite XLS, hydrogels, thermoresponsive hydrogel.

\section{INTRODUCCIÓN}

Las 2-oxazolinas son sustancias heterocíclicas, que polimerizan vía una polimerización catiónica por apertura de anillo, la cual es iniciada por grupos electrófilos. La polimerización de las 2-oxazolinas transcurre en una forma "viva", y se pueden predeterminar, desde el inicio de la polimerización, el peso molecular del polímero y su distribución así como su funcionalidad $^{1-3}$.

Con las 2-oxazolinas se pueden elaborar macromonómeros, copolímeros en bloque e injertados ${ }^{2,3}$. La polimetil- y polietil-oxazolina, tienen un carácter hidrofílico.

Los hidrogeles tienen como propiedad principal la de absorber agua en relativamente grandes cantidades pero al hacerlo pierden estabilidad mecánica. Haraguchi y colaboradores por primera vez utilizaron la arcilla Laponita XLS como elemento entrecruzador de las cadenas de poli(NiPAAm) para obtener hidrogeles termoconmutables y con buenas propiedades mecánicas $^{4,5}$. Sin embargo, al intentar conseguir una sensibilidad al $\mathrm{pH}$ mediante la 
introducción al sistema de polimerización anterior del ácido acrílico no tuvo éxito ya que los grupos ácidos formaban complejos con las placas de Laponita y se perdían las buenas propiedades mecánicas y la transparencia.

En la presente investigación se utilizó la estrategia de elaborar primero el hidrogel de poli(NiPAAm) conteniendo grupos protectores ésteres (provenientes de los macromonómeros de 2-oxazolinas) y después hidrolizar estos, in situ, en el hidrogel ya formado, para obtener los grupos ácido carboxílico dentro de su estructura y evitar así, en lo posible, la formación de complejos entre los grupos ácido y la Laponita XLS.

\section{PARTE EXPERIMENTAL}

Las sustancias utilizadas en esta investigación fueron obtenidas de las firmas Aldrich y Merck y fueron purificadas según métodos estándar de la literatura ${ }^{3,6}$. La 2-metil- y 2-(2-metoxicarboniletil)-2-oxazolina fueron sintetizadas en el laboratorio mediante los procedimientos descritos en la literatura ${ }^{6}$. Los macromonómeros de 2-oxazolinas fueron elaborados según método descrito en publicación anterior ${ }^{3}$.

Síntesis de los hidrogeles utilizando Laponita XLS como entrecruzador: Procedimiento típico (Ej. Hidrogel HGL-3): En un vaso de $50 \mathrm{~mL}$, se disolvieron 1,0 g $(8,8 \mathrm{mmol}) \mathrm{de}$ NiPAAm, 0,2 g (0,10 mmol) de macromonómero M1 sin hidrolizar, 0,2 g de Laponita XLS, 0,030 g de TEMED en $4 \mathrm{~mL}$ de agua hasta disolución total. Luego, esta mezcla se colocó en un recipiente cerrado y se burbujeó nitrógeno gaseoso durante 15 minutos. Posteriormente, el sistema fue cerrado y colocado en un baño de agua con hielo $\left(5^{\circ} \mathrm{C}\right)$ durante 15 minutos. En forma paralela, en otro vaso de $25 \mathrm{~mL}$ se disolvió $0,025 \mathrm{~g}$ de APS en $1 \mathrm{~mL}$ de agua y se burbujeó nitrógeno por 15 minutos. A continuación, se agregó la solución de APS a la primera solución y se mezcló bien. La reacción siguió su curso en el baño de agua fría hasta la formación de un hidrogel.

La purificación del hidrogel se realizó mediante lavado con agua destilada. Para esto el hidrogel fue colocado en un litro de agua destilada por 24 horas para eliminar restos de monómeros y de iniciador que no hubieran reaccionado, durante este período el agua fue cambiada cuatro veces. Finalizada la purificación, el hidrogel fue secado a $30^{\circ} \mathrm{C}$ hasta peso constante y guardado en un desecador. El hidrogel fue caracterizado por espectroscopia RMN, mediante la técnica HR-MAS (giro de ángulo mágico de alta resolución).

Para la síntesis de otros hidrogeles, el procedimiento descrito anteriormente fue el mismo solo que se varió las cantidades de los componentes. En la tabla 1 de la discusión de resultados se exponen las composiciones para todos los hidrogeles elaborados.

Hidrólisis de los hidrogeles: Procedimiento típico (Ej. Hidrogel H-HGL5): En un vaso de $250 \mathrm{~mL}$, se agregó $1,0 \mathrm{~g}$ de hidrogel y se adicionó cuatro equivalentes gramos de una solución de $\mathrm{NaOH} 0,1 \mathrm{~N}$ en agua, todo este sistema se calentó a $40^{\circ} \mathrm{C}$ por 48 horas. Pasado 
este tiempo, el sistema fue enfriado y neutralizado con solución $\mathrm{HCl} 0,1 \mathrm{~N}$ hasta un $\mathrm{pH}=5,6$, aproximadamente. Después de la neutralización, se purificó el hidrogel mediante lavado con agua destilada. Para esto los hidrogeles fueron sumergidos en un balón de un litro de agua durante 48 horas donde cada 12 horas se cambió el agua destilada. Terminado este tiempo, el agua contenida en el hidrogel fue eliminada mediante calentamiento a $32^{\circ} \mathrm{C}$ hasta obtener un hidrogel seco.

El hidrogel hidrolizado H-HGL5 fue caracterizado estructuralmente por su espectro de resonancia magnética nuclear de hidrógeno.

Espectroscopía RMN: Los espectros ${ }^{1} \mathrm{H}-\mathrm{y}{ }^{13} \mathrm{C}-\mathrm{RMN}$ fueron obtenidos en un equipo RMN Avance III 500 (Bruker, Alemania) a $500 \mathrm{MHz}$ para $1 \mathrm{H}$ y $125,7 \mathrm{MHz}$ para $13 \mathrm{C}$.

Prueba de absorción de agua: Se colocó en un vaso una porción de hidrogel hidrolizado seco (peso inicial=Ws) en $80 \mathrm{~mL}$ de agua destilada a temperatura ambiente durante 24 horas. Luego de este tiempo se extrajo el hidrogel hinchado en agua y se pesó (Wh). El grado de absorción de agua del hidrogel fue calculado mediante la ecuación: $\mathrm{Q}=(\mathrm{Wh}-\mathrm{Ws}) / \mathrm{Ws}$, donde Q es el grado de absorción de agua del hidrogel (g H2O/g de HG seco).

Prueba de sensibilidad a la temperatura: Se determinó el grado de contracción del volumen de los hidrogeles al aumentar la temperatura. Se asumió que el peso del hidrogel fue siempre proporcional a su volumen. El peso del hidrogel a $25^{\circ} \mathrm{C}$ es proporcional al volumen inicial del hidrogel. El porcentaje de contracción, en respuesta al aumento de temperatura, se determinó mediante la ecuación: \% Contracción $=100 *\left(\mathrm{~W}_{\text {inicial }}-\mathrm{Wf}_{\text {inal }}\right) / \mathrm{W}_{\text {inicial }}$

Procedimiento: En un vaso se colocó una porción del hidrogel en $80 \mathrm{~mL}$ de agua a temperatura ambiente por 24 horas. Pasado este tiempo, se sacó el hidrogel del agua y se determinó su peso $\left(\mathrm{W}_{\text {inicial }}\right)$. Seguidamente, la misma porción de hidrogel se sumergió nuevamente en un recipiente con agua y este fue colocado en un baño termostatizado a una temperatura determinada durante 60 minutos; luego, el hidrogel nuevamente se sacó del agua y se pesó $\left(\mathrm{W}_{\text {final }}\right)$. Se determinó el peso remanente del hidrogel a esta temperatura mediante la ecuación: Peso remanente $(\%)=100 *\left(\mathrm{Peso}_{\text {final }} / \mathrm{Peso}_{\text {inicial }}\right)$. La contracción del hidrogel es la diferencia entre el peso inicial y el peso remanente. Para cada temperatura, se obtuvo un peso remanente $(\%)$ del hidrogel y estos valores fueron ploteados versus la temperatura $\left({ }^{\circ} \mathrm{C}\right)$.

Prueba de sensibilidad al pH: Procedimiento típico: En un vaso de $100 \mathrm{~mL}$, se sumergió una porción de hidrogel hidrolizado en agua a $\mathrm{pH}=6,5$ a la temperatura ambiente $\left(20^{\circ} \mathrm{C}\right)$ por 24 horas hasta alcanzar el equilibrio. Luego se pesó el hidrogel, este peso fue denominado "Peso inicial $\left(\mathrm{W}_{\text {inicial }}\right)$ ". Seguidamente, se procedió a ajustar el $\mathrm{pH}$ del medio a un $\mathrm{pH}=1$ agregando para esto solución de $\mathrm{HCl}, \mathrm{pH}=1$, dejando nuevamente el hidrogel sumergido por 24 horas en este medio ácido. Cuando el equilibrio fue alcanzado, el hidrogel fue extraído y pesado, a este peso se le denominó "Peso final $\left(\mathrm{W}_{\text {final }}\right)$ ". Para ensayar la sensibilidad al $\mathrm{pH}$ básico $(\mathrm{pH}=10)$, se repite el procedimiento anteriormente descrito, pero agregando solución de $\mathrm{pH}=10$. La variación del volumen que mostró el hidrogel en respuesta al cambio del 
$\mathrm{pH}$, ácido o básico, fue determinado mediante la relación: $\%$ Contracción $=100 *\left(\mathrm{Peso}_{\text {inicial }^{-}}\right.$ $\left.\left.\mathrm{Peso}_{\text {final }}\right) / \mathrm{Peso}_{\text {inicial }}\right)$.

Análisis por microscopía electrónica de transmisión (TEM): Las muestras fueron analizadas por la Química Sra. Ute Reuter del Instituto Leibniz de Investigaciones en Polímeros de Dresden, Alemania, aplicando el procedimiento estándar. Microscopio TEM: Marca: Zeiss, Modelo: Libra 120, Resolución máxima: 0,4 nm.

\section{DISCUSIÓN DE RESULTADOS}

Síntesis de los macromonómeros: Como fue descrito en una publicación anterior ${ }^{3}$ la síntesis de los macromonómeros M1 y M2 fue realizada a partir de la polimerización catiónica por apertura de anillo de la 2-metil-2-oxazolina (MeOXA) y la 2-(2-metoxicarboniletil)2-oxazolina (ESTER-OXA) iniciada por el clorometilestireno (CMS) en presencia del catalizador ioduro de sodio. Debido a la iniciación por el CMS, el grupo vinilo fue introducido en el extremo de la cadena polimérica desde el inicio de la polimerización.

La reacción se realizó a una temperatura relativamente baja, de $78^{\circ} \mathrm{C}$, durante siete horas y con una baja concentración del iniciador en el medio de reacción. Esto se realizó así para evitar una polimerización prematura del grupo vinilo del CMS. Sin embargo, estas condiciones de reacción sí fueron suficientes para conseguir la polimerización completa de las 2-oxazolinas. Se usó como solvente el acetonitrilo. Bajo estas condiciones de reacción, los macromonómeros M1 y M2 fueron obtenidos con pesos moleculares de 2220 y 3023 (o grados de polimerización de 20 y 24 , respectivamente) y con un contenido de grupos ésteres de 23 y $49 \mathrm{~mol} \%$, respectivamente. Se obtuvieron rendimientos de polimerización cercanos al $100 \%$. La reacción de polimerización se muestra en el esquema 1.

La polimerización fue favorecida por tres factores: la mayor nucleofilicidad de las oxazolinas respecto del ión ioduro, la tensión en el anillo oxazolínico, y la gran estabilidad del grupo amida resultante de la misma. Por ello, la reacción procedió vía el mecanismo iónico, reaccionando las cadenas propagantes solo con los monómeros de 2-oxazolinas y la reacción se llevó a cabo de forma "viva", es decir, ocurrió sin reacciones de terminación o de transferencia de cadena. 


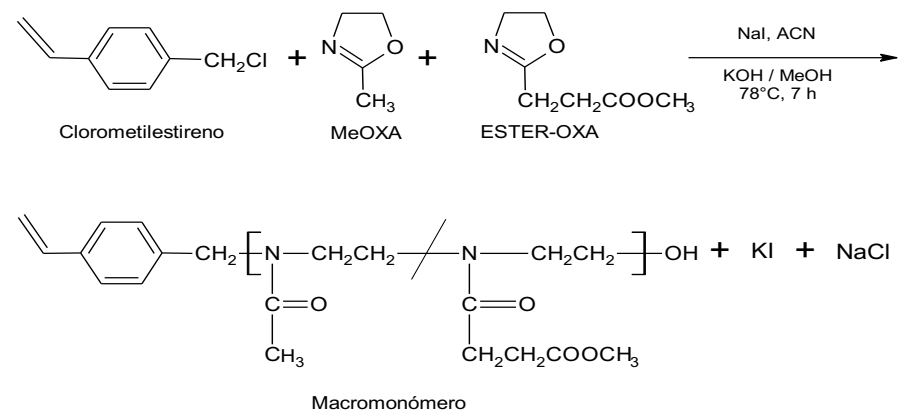

Esquema 1. Síntesis de los macromonómeros

El macromonómero M1 fue caracterizado estructuralmente mediante sus espectros de resonancia magnética nuclear de hidrógeno y carbono (ver 1H RMN del macromonómero M1 hidrolizado en figura 1). Para M2 el espectro fue similar. Todas las mediciones se realizaron a $30^{\circ} \mathrm{C}$ utilizando metanol deuterado (CD3OD).

${ }^{13} \mathrm{C}-\mathrm{RMN}\left(\mathrm{CD}_{3} \mathrm{OD}\right) \delta: 21,3-21,8\left(\mathrm{C}(\mathrm{O}) \mathrm{CH}_{3}\right) ; 28,5\left(\mathrm{C}(\mathrm{O}) \underline{\mathrm{CH}}_{2}\right) ; 30\left(\underline{\mathrm{CH}}_{2} \mathrm{C}(\mathrm{O}) \mathrm{OCH}_{3}\right) ; 44-48$ $\left(\mathrm{NCH}_{2} \mathrm{CH}_{2}\right) ; 52,2\left(\mathrm{C}(\mathrm{O}) \mathrm{OCH}_{3}\right) ; 54,1\left(\mathrm{Ar}-\mathrm{CH}_{2}\right) ; 61\left(\mathrm{CH}_{2} \mathrm{OH}\right) ; 115\left(\mathrm{CH}_{2}=\right) ; 125-131\left(\mathrm{CH}_{\mathrm{ar}}\right)$; $137-140\left(\mathrm{C}_{\mathrm{ar}}\right) ; 137,5(-\mathrm{CH}=) ; 173,6\left(\underline{\mathrm{COCH}}_{3} \mathrm{y} \underline{\mathrm{COCH}}_{2}\right) ; 175,1\left(\underline{\mathrm{C}}(\mathrm{O}) \mathrm{OCH}_{3}\right)$.

El espectro ${ }^{1} \mathrm{H}-\mathrm{RMN}$ de $\mathrm{M} 1$ tiene una señal a 3,6 ppm que corresponde al metilo del grupo éster del monómero ESTER-OXA. Posteriormente, esta señal desapareció al realizarse la hidrólisis de los grupos éster y se formaron grupos ácido carboxílicos dentro del hidrogel (Fig. 1). Asimismo, la señal a 52,2 ppm del espectro ${ }^{13} \mathrm{C}-\mathrm{RMN}$ correspondiente al átomo de carbono del grupo metilo del éster desapareció al ser hidrolizado el hidrogel, indicando esto que la hidrolisis fue cuantitativa $(100 \%)$.

La presencia en los espectros RMN de las señales correspondientes a los diferentes tipos de protones y átomos de carbono de la estructura teórica del macromonómero, confirman que ocurrió la formación del copolímero a partir de los monómeros MeOXA y ESTER-OXA.

Del análisis cuantitativo del espectro $1 \mathrm{H}-\mathrm{RMN}$ se determinó el grado de polimerización de M1 y M2, que fue de 20 y 24, respectivamente, y también el porcentaje molar de ESTEROXA y MeOXA en los macromonómeros. Los valores fueron determinados a partir de la comparación de las integrales de las señales de los hidrógenos metilénicos de $\mathrm{COCH}_{2} \mathrm{CH}_{2} \mathrm{COO}$ (f, g, 2,4-2,8 ppm) del ESTER-OXA, de la integrales de la señal del metilo $\mathrm{CH}_{3} \mathrm{CO}$ (e, 2,11 ppm) del MeOXA y de los protónes del grupo iniciante CMS (a, a', b, Har, 5,25; 5,8; 6,75 y 7,1-7,5 ppm). Los porcentajes molares calculados de ESTER-OXA fueron de $23 \%$ y $49 \%$ para los macromonómeros M1 y M2, respectivamente. Estos resultados confirman que en la polimerización no hubo reacciones secundarias de transferencia de cadena o terminación, es decir, la polimerización se realizó en forma "viva" y sin la polimerización prematura de los grupos vinilo de los macromonómeros. 


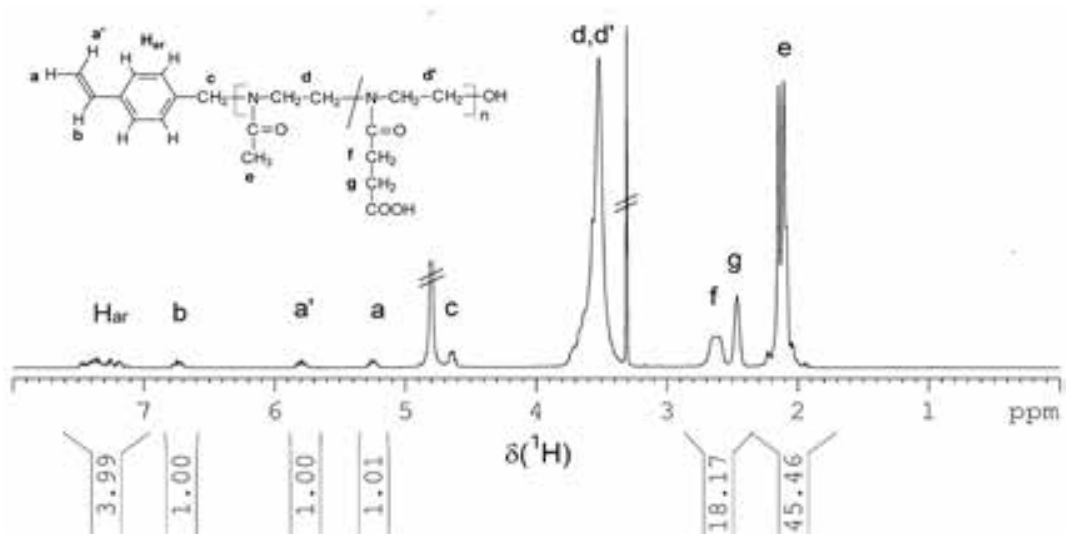

Figura 1. Espectro ${ }^{1} \mathrm{H}-\mathrm{RMN}$ del macromonómero hidrolizado $\mathrm{H}-\mathrm{M} 1$ a $30^{\circ} \mathrm{C}$ en

CD3OD.

\section{Síntesis de los hidrogeles}

El objetivo de sintetizar hidrogeles basados en copolímeros de NiPAAm y macromonómeros de 2-oxazolinas utilizando Laponita XLS como agente entrecruzante fue el de obtener materiales que sean sensibles a la temperatura y al cambio de $\mathrm{pH}$ del medio, y que a la vez posean mejores propiedades mecánicas que los hidrogeles convencionales que utilizan un agente entrecruzante orgánico.

La estrategia seguida fue sintetizar primero los hidrogeles con los grupos éster de los macromonómeros contenidos en su estructura y luego, recién en un siguiente paso, hidrolizar los grupos éster para obtener grupos ácidos. Esta estrategia difiere de la de Haraguchi ${ }^{4}$ et al., quien sintetizó hidrogeles a partir de N-isopropilacrilamida (NiPAAm), ácido acrílico y Laponita lo que condujo a una estructura heterogénea con pobres propiedades mecánicas de los mismos. Esto se atribuyó a la formación de complejos entre el ácido acrílico y la superficie de la Laponita, cargada negativamente.

Los hidrogeles fueron obtenidos mediante la copolimerización por radicales libres de la $\mathrm{N}$-isopropilacrilamida (NiPAAm), macromonómeros de 2-oxazolina (M1 o M2 sin hidrolizar) en presencia de Laponita XLS. La polimerización se realizó en medio acuoso y fue iniciada por un sistema de iniciación redox basado en persulfato de amonio (APS) y catalizador N,N,N',N'-tetrametiletilendiamina (TEMED) (esquema 2).

Se obtuvieron hidrogeles con buena consistencia mecánica y transparencia y que contenían grupos éster. La velocidad de la reacción fue alta, produciendo hidrogeles en solo 10 minutos. Los detalles experimentales y resultados obtenidos en la síntesis de los hidrogeles se resumen en la tabla 1. 
Tabla 1. Síntesis de hidrogeles con Laponita XLS

(datos experimentales y resultados)

\begin{tabular}{|c|c|c|c|c|c|}
\hline $\mathrm{HGL}^{\text {a) }}$ & $\begin{array}{l}\text { NiPAAm }{ }^{b)} \\
\quad(g)\end{array}$ & $\begin{array}{l}\mathrm{MM}^{\mathrm{c})} \\
(\mathrm{g})\end{array}$ & $\begin{array}{l}\mathrm{LAP}^{\mathrm{d})} \\
(\mathrm{g})\end{array}$ & NiPAAm/MM ${ }^{\mathrm{e}}$ & $\begin{array}{c}\mathrm{R}^{\mathrm{f})} \\
(\%)\end{array}$ \\
\hline HGL-1 & 1,0 & 0 & 0,2 & - & 88 \\
\hline HGL-2 & 1,0 & 0,20 & 0,2 & 98,3 & 75 \\
\hline HGL-3 & 1,0 & 0,10 & 0,2 & 196,6 & 86 \\
\hline HGL-4 & 1,0 & 0,05 & 0,2 & 393,2 & 82 \\
\hline HGL-5 & 1,0 & 0,40 & 0,2 & 59,1 & 82 \\
\hline HGL-6 & 1,0 & 0,10 & 0,2 & 236,3 & 88 \\
\hline
\end{tabular}

Donde:

${ }^{a}$ Hidrogel sintetizado, ${ }^{b}$ NiPPAm: N-isopropilacrilamida, ${ }^{\mathrm{c}} \mathrm{MM}$ : Macromonómero, ${ }^{\mathrm{d}}$ LAP: Laponita XLS, ${ }^{\mathrm{e}}$ Relación molar inicial de monómeros de NiPAAm/MM, ${ }^{\mathrm{f}}$ Rendimiento $(\%)=100 *$ (Peso HGL obtenido/ $\Sigma$ Pesos de reactantes (NiPAAm, macromonómero y Laponita)), En los hidrogeles HGL-2, -3,y -4 se usó el macromonómero M1 (con 23\% de Esteroxa y $\mathrm{PD}=20$ ) y en los hidrogeles HGl-5, -6 se usó el macromonómero M2 (con un $49 \%$ molar de Esteroxa y PD=24). En el hidrogel HGL-1 no se usó macromonómero. ${ }^{h}$ El sistema iniciador fue APS: Persulfato de amonio, y TEMED: N,N,N',N'tetrametiletilendiamina. En todos los experimentos se usó 0,025 gramos de APS y 0,030 gramos de Temed y $5 \mathrm{~mL}$ de agua como solvente.

Como producto de la polimerización se obtuvo un hidrogel conteniendo meros de NiPAAm y macromonómeros de MeOXA y ESTER-OXA. Estas cadenas poliméricas estarían adheridas a las plaquetas de Laponita XLS que funcionarían como agente entrecruzador. Los rendimientos obtenidos estuvieron entre 75 y $97 \%$ (tabla 1 ).

Los hidrogeles fueron caracterizados estructuralmente vía la técnica RMN-HR-MAS (high resolution magic angle spinning). Todas las mediciones se realizaron a $30^{\circ} \mathrm{C}$ utilizando agua deuterada $\left(\mathrm{D}_{2} \mathrm{O}\right)$.

En la figura 2 se muestra el espectro ${ }^{1} \mathrm{H}-\mathrm{RMN}$ (técnica HR-MAS) para el hidrogel HGL-5. A continuación se indican las asignaciones de las señales observadas. Cabe señalar que los espectros para todos los hidrogeles elaborados fueron similares.

${ }^{1} \mathrm{H}-\mathrm{RMN}-\mathrm{HR}-\mathrm{MAS}\left(\mathrm{D}_{2} \mathrm{O}\right) \delta: 1,15-1,25\left(\mathrm{CH}\left(\mathrm{CH}_{3}\right)_{2}\right) ; 1,4-2,2\left(\mathrm{CH}_{2} \mathrm{CH}\right) ; 2,1-2,2\left(\mathrm{CH}_{3} \mathrm{CO}\right)$; 2,45-2,8 (C(O) $\left.\underline{\mathrm{H}}_{2} \underline{\mathrm{C}}_{2} \mathrm{C}(\mathrm{O}) \mathrm{OCH} 3\right) ; 3,3-3,6(\mathrm{NCH} 2 \mathrm{CH} 2) ; 3,71$ (C(O)OCH3); 3,9-4,0 (CH). 


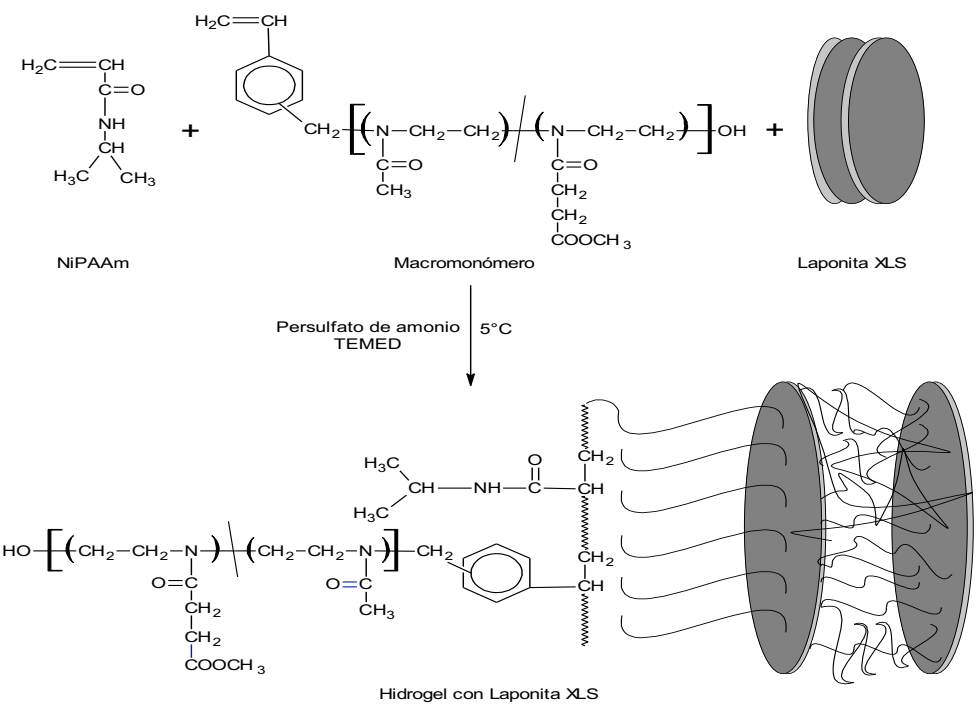

Esquema 2. Síntesis de hidrogeles con Laponita XLS.

La presencia, en los espectros ${ }^{1} \mathrm{H}-\mathrm{RMN}$, de las señales correspondientes a los diferentes tipos de protones del NiPAAm y macromonómero demuestran cualitativamente que ocurrió la formación del hidrogel mediante entrecruzamiento con Laponita XLS.

La señal a 3,7 ppm en los espectros 1H-RMN de los hidrogeles, corresponde al grupo metilo del éster del monómero ESTER-OXA. Posteriormente, cuando se realizó la hidrólisis de los hidrogeles, esta señal desapareció porque los grupos éster pasaron a ser grupos ácido carboxílicos.
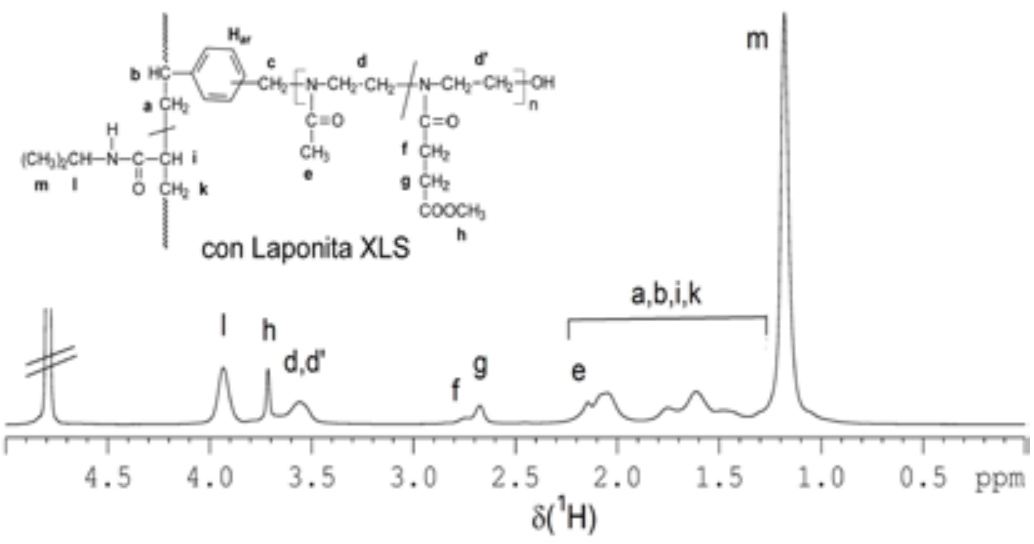

Figura 2. Espectro ${ }^{1} \mathrm{H}-\mathrm{RMN}$ - (técnica HR-MAS) del hidrogel HGL-5 a $30^{\circ} \mathrm{C}$ en $\mathrm{D}_{2} \mathrm{O}$ 
Hidrólisis de los hidrogeles: La hidrólisis de los hidrogeles fue llevada a cabo en medio básico diluido $(0,1 \mathrm{~N} \mathrm{NaOH})$ y a temperatura relativamente baja $\left(45^{\circ} \mathrm{C}\right)$ para hidrolizar selectivamente los grupos éster a grupos ácido carboxílico y evitar la hidrólisis de los grupos amida. Como producto de la hidrólisis, se obtuvo un hidrogel transparente y con buena consistencia mecánica. A continuación se muestran las señales obtenidas para el hidrogel hidrolizado H-HGL2.

${ }^{1} \mathrm{H}-\mathrm{RMN}\left(\mathrm{D}_{2} \mathrm{O}\right) \delta: 1,15-1,25\left(\mathrm{CH}\left(\mathrm{CH}_{3}\right)_{2}\right) ; 1,4-2,2\left(\mathrm{CH}_{2} \mathrm{CH}\right) ; 2,1-2,2\left(\mathrm{CH}_{3} \mathrm{CO}\right) ; 2,45-2,7$ $\left(\mathrm{C}(\mathrm{O}) \underline{\mathrm{H}}_{2} \underline{\mathrm{C}}_{2} \mathrm{COOH}\right) ; 3,3-3,8\left(\mathrm{NCH}_{2} \mathrm{CH}_{2}\right) ; 3,9-4,0(\mathrm{CH})$.

Los resultados indican que ocurrió un $100 \%$ de hidrólisis ya que la señal h a 3,71 ppm del grupo éster $\left(\mathrm{CH}_{3} \mathrm{O}\right)$ ha desaparecido completamente en el espectro (Fig. 3, espectro a $25^{\circ} \mathrm{C}$ ).

\section{Análisis de hidrogeles por microscopía electrónica de transmisión (TEM)}

La caracterización morfológica, del hidrogel hidrolizado H-HGL2, mediante microscopía electrónica de transmisión (TEM) (figura 4, HGL-2) mostró que la distribución de las plaquetas de Laponita XLS fue homogénea dentro del hidrogel, formando una red entrecruzada. Esto es similar a lo encontrado por Haraguchi ${ }^{4}$ et.al.

Estabilidad mecánica de los hidrogeles: Los hidrogeles mostraron una buena estabilidad mecánica. Mediante un ensayo cualitativo, se estiró el hidrogel HGL-2 (sin hidrolizar) mostrando una gran capacidad elástica, se alargó más del $50 \%$ de su dimensión (figura 5). Estas remarcables mejoras en las propiedades mecánicas de los hidrogeles elaborados pueden ser atribuidas a su estructura entrecruzada homogénea de polímero-arcilla inorgánica.

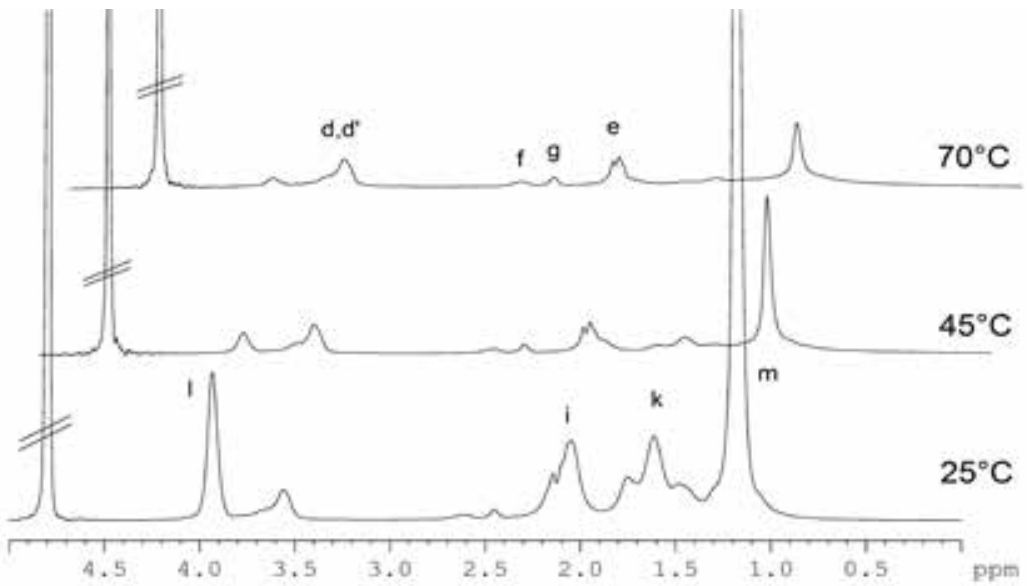

Figura 3. Espectros ${ }^{1} \mathrm{H}-\mathrm{RMN}$ - (técnica HR-MAS) del hidrogel hidrolizado H-HGL2 a diferentes temperaturas en $\mathrm{D}_{2} \mathrm{O}$. 


\begin{abstract}
Absorción de agua: los hidrogeles hidrolizados H-HGL2, H-HGL3 y H-HGL4, todos con macromonómero M1 (23\% ESTER-OXA), mostraron que la absorción de agua se incrementa cuando aumenta la relación molar macromonómero (MM)/NiPAAm (los valores de absorción de agua para H-HGL2, H-HGL-3, y H-HGL-4 son 38,5, 16,5, y 12,5 g agua/g hidrogel seco, respectivamente). Este resultado es debido a la mayor hidrofilicidad de los grupos carboxilatos y carboxilos, contenidos en el macromonómero.
\end{abstract}

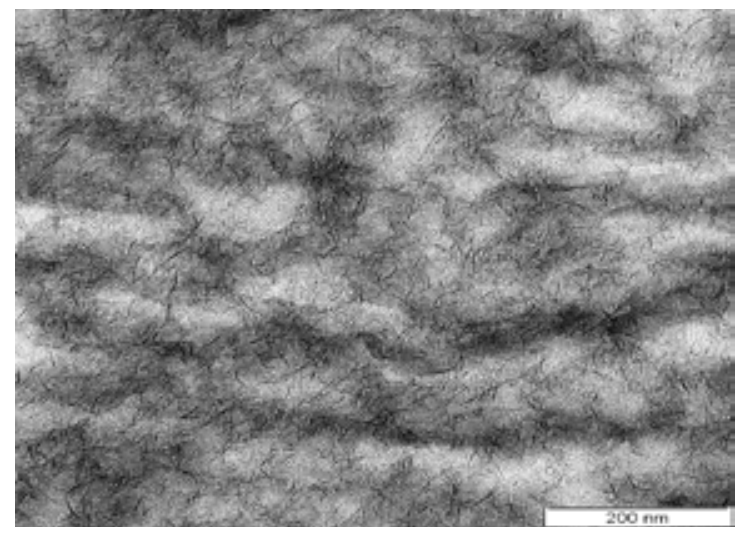

Figura 4. Microscopía electrónica de transmisión (TEM) para el hidrogel hidrolizado H-HGL2 con una magnificación de 40,000X.

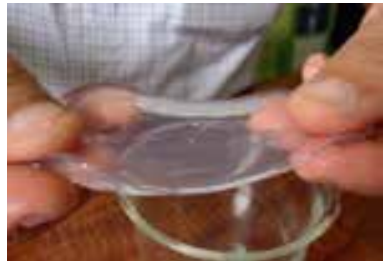

Antes del estiramiento

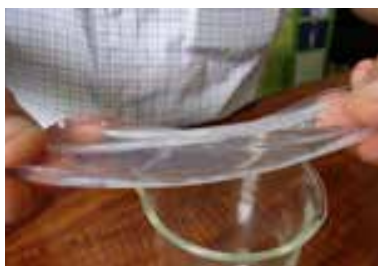

Después del estiramiento

Figura 5. Hidrogel HGL-2 (sin hidrolizar) con buena consistencia mecánica.

En el caso de los hidrogeles H-HGL5 y H-HGL-6 los valores de absorción de agua fueron 43 y 33,3 g agua/g de hidrogel seco. La absorción de agua del primero de ellos fue mayor debido a que contiene mayor cantidad de macromonómero M2, el cual es aún mas hidrofilico que M1 debido a que contiene más grupos ácido carboxílicos (49 vs $23 \%$ ).

Sensibilidad a la temperatura: Los hidrogeles presentaron sensibilidad a la temperatura. Esta sensibilidad se expresó como una contracción del volumen del hidrogel al aumentar la temperatura. Debido a la forma irregular del hidrogel no fue posible medir su volumen, por eso se midió el peso del hidrogel y se asumió que este fue siempre proporcional al volumen del mismo. En la figura 6 se observa la variación del peso de los hidrogeles al aumentar la temperatura. 
En todos los casos hubo una contracción del hidrogel al aumentar la temperatura aunque con las muestras H-HGL-5 y H-HGL-6 hubo primero una expansión y luego una contracción. El hidrogel HHGL-1 solo contiene poliNiPAAm y se contrae a una temperatura menor a los demás (transición conformacional a $35^{\circ} \mathrm{C}$ ) debido a que contiene menor hidrofilicidad por no contener macromonómero. En los demás hidrogeles a mayor relación molar NiPAAm/ macromonómero dentro del hidrogel, la contracción es mayor y ocurre de forma más rápida debido a que existe mayor cantidad del elemento termosensible (poliNiPAAm) en el mismo. $\mathrm{Al}$ aumentar el contenido de macromonómero, la temperatura a la cual ocurre la contracción (transición conformacional) se incrementa debido a que aumenta la hidrofilicidad del hidrogel por el contenido de macromonómero $y$, asimismo, se observa que la transición disminuye en intensidad.

Los hidrogeles H-HGL5 y H-HGL6 tienen un relativo alto contenido de grupos ácido carboxílicos y carboxilatos (provenientes de la gran cantidad del macromonómero M2 usada en su síntesis), por esto poseen una fuerte hidrofilicidad y a mayor temperatura esta fuerza es mayor que la fuerza de contracción del NiPAAm haciendo que los hidrogeles primero se expandan hasta un punto para luego empezar a contraerse.

En la figura 3 se muestran los espectros ${ }^{1} \mathrm{H}-\mathrm{RMN}$ para el hidrogel hidrolizado H-HGL2 a temperaturas de $25^{\circ} \mathrm{C}, 45^{\circ} \mathrm{C}$ y $70^{\circ} \mathrm{C}$ en $\mathrm{D} 2 \mathrm{O}$. Se observa claramente que a medida que la temperatura aumenta disminuyen en intensidad las señales i - $\mathrm{m}$ correspondientes a los segmentos del poliNiPAAm contenidos en los hidrogeles debido al colapso de estas cadenas poliméricas. En tanto que las cadenas laterales de polioxazolinas muestran buena movilidad incluso a $70^{\circ} \mathrm{C}$ y por esto se pueden observar en el espectro (señales d-g).

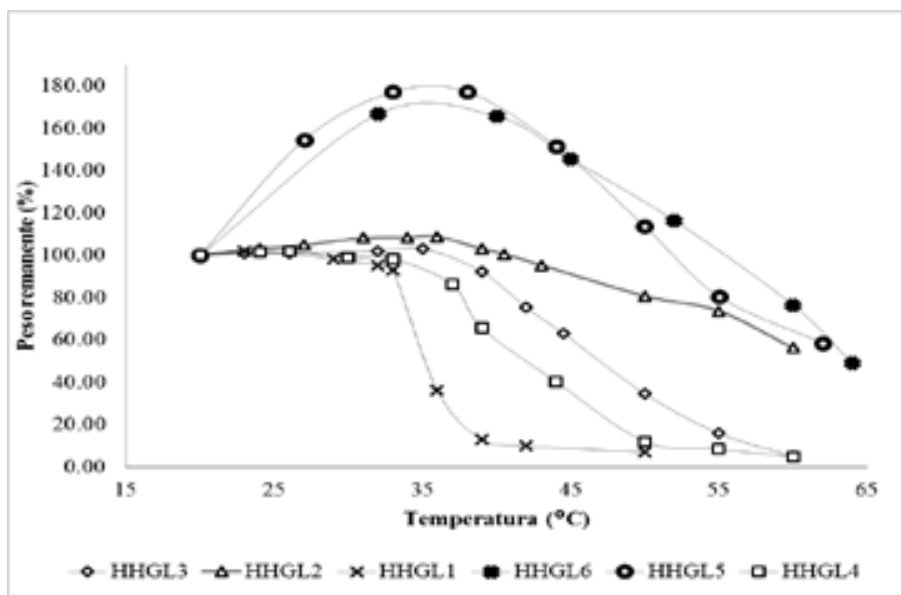

Figura 6. Temperatura $\left({ }^{\circ} \mathrm{C}\right)$ versus peso remanente $(\%)$ de los hidrogeles. 
Prueba de sensibilidad al pH: Debido a su contenido de grupos ácido carboxílicos, provenientes del macromonómero de 2-oxazolina, los hidrogeles mostraron sensibilidad al pH del medio. Para la interpretación de los resultados, hemos tomado como referencia que el hidrogel al $\mathrm{pH}=6,5$ tiene un volumen inicial que asumimos corresponde al $100 \%$, y el cambio de volumen a diferentes $\mathrm{pH}\left(\begin{array}{l}1 \\ \text { y }\end{array} 10\right)$ se refieren a ese porcentaje inicial. La contracción o expansión del volumen fue evaluada mediante la pérdida o ganancia de peso del hidrogel.

Fueron evaluados algunos hidrogeles. Los hidrogeles H-HGL2 y H-HGL-3 se contrajeron 35 y $32 \%$ a un $\mathrm{pH}=1$, respectivamente. En tanto que a un $\mathrm{pH}=10$ estos mismos hidrogeles se contrajeron 16 y $35 \%$, respectivamente.

A pH 6,5 (pH del agua destilada utilizada) el hidrogel esta hinchado en agua y el hidrogel hidrolizado absorbe agua debido a la polaridad por la disociación parcial de los grupos ácido carboxílicos. A un $\mathrm{pH}$ bajo $(\mathrm{pH}=1)$ el hidrogel se contrae debido posiblemente por la formación de enlaces de hidrógeno entre los grupos ácidos y los grupos amida dentro del hidrogel. A un $\mathrm{pH}$ intermedio el hidrogel se expande debido a la repulsión electrostática entre los grupos carboxilato de sodio. A un $\mathrm{pH}$ más elevado, como $\mathrm{pH}=10$, el hidrogel debería seguir expandiéndose pero no ocurre esto y el hidrogel se contrae de nuevo, no hemos encontrado una explicación para este fenómeno

\section{CONCLUSIONES}

Es posible elaborar hidrogeles mediante la copolimerización radicalar de $\mathrm{N}$-isopropilacrilamida (NiPAAm) y macromonómeros de 2-oxazolinas en medio acuoso en presencia de la arcilla Laponita XLS e iniciada por APS/TEMED. Los macromonómeros utilizados, M1 y M2, tuvieron un porcentaje molar de monómero ESTER-OXA de $23 \%$ y $49 \%$ y grados de polimerización 20 y 24 , respectivamente.

El grado de absorción de agua de los hidrogeles fue directamente proporcional al contenido de los macromonómeros hidrolizados de 2-oxazolinas en los mismos.

Los hidrogeles sintetizados presentaron buenas propiedades mecánicas comparadas con los hidrogeles convencionales. La Laponita XLS funciona en estos sistemas de polimerización como un elemento entrecruzador y de refuerzo mecánico. El hidrogel fue caracterizado morfológicamente mediante el ensayo de microscopía electrónica de transmisión (TEM) y se demostró que la Laponita estaba homogéneamente dispersa en la red del hidrogel contribuyendo esto a la estabilidad mecánica.

Los hidrogeles presentaron sensibilidad a la temperatura debido a los segmentos de PoliNiPAAm, esta sensibilidad se manifiesta mediante una contracción de su volumen al aumentar la temperatura. La temperatura de transición, que es el valor medio del rango de temperatura, aumenta al incrementarse en contenido del macromonómero. 
La sensibilidad al pH de los hidrogeles fue evidenciada mediante una contracción del hidrogel al elevarse o disminuirse el $\mathrm{pH}$ del medio respecto del $\mathrm{pH}$ neutro. La contracción del hidrogel a pH ácido es probablemente causada por los enlaces de hidrógeno entre la amida y los grupos ácidos provenientes del macromonómero dentro del hidrogel. A pH alto, la contracción puede estar relacionada a la alta carga iónica total dentro del hidrogel.

\section{AGRADECIMIENTOS}

Al Fondo para la Innovación, Ciencia y Tecnología (Fincyt), a la Pontificia Universidad Católica del Perú (PUCP) y al Instituto Leibniz de Investigaciones en Polímeros de Dresden (IPF), Alemania por el financiamiento de esta investigación mediante el proyecto FINCYT212-2013-IA ("hidrogeles actuadores con doble sensibilidad y su aplicación en transistores químicos").

\section{REFRERENCIAS BIBLIOGRÁFICAS}

1. Aoi K, Okada M. Polymerization of oxazolines. Prog Polym Sci. 1996; 21: 151-208.

2. Kobayashi S, Saegusa T. Block and graft copolymers of 2-oxazolines. Macromol Chem Phys.1985; 12: 11-24.

3. Rueda J, Campos E, Komber H, Zschoche S, Voit B. Synthesis and characterization of new $\mathrm{pH}$ - and thermo-responsive hydrogels based on $\mathrm{N}$-isopropylacrylamide and 2-oxazolines. Des Monomers Polym. 2014; 17: 208-216.

4. Haraguchi K, Takehisa T. Nanocomposite Hydrogels: A Unique Organic-Inorganic Network Structure with Extraordinary Mechanical, Optical, and Swelling/De-swelling Properties. Adv Mater. 2002; 14: 1120-1124.

5. Schild HG. Poly(N-isopropylacrylamide): experiment, theory and application. Prog Polym Sci. 1992; 17: 163-240.

6. Witte H, Seeliger W. Cyclische Imidsäureester aus Nitrilen und Aminoalkoholen. Liebigs Ann Chem. 1974; 1974(6): 996-1009. 